\title{
Abandono do tratamento da fuberculose: uma revisão integrativa
}

\section{Abandonment of tuberculosis treatment: an integrative review}

\section{Melisane Regina Lima Ferreira', Rafaele Oliveira Bonfim², Tatiane Cabral Siqueira², Nathalia Halax Orfão}

\footnotetext{
'Autora para correspondência. Universidade Federal de Rondônia. Porto Velho, Rondônia, Brasil. melisane1206@gmail.com ${ }^{2}$ Universidade Federal de Rondônia. Porto Velho, Rondônia, Brasil. rafaelebonfim@hotmail.com, tatianecabralsiqueira@gmail.com, nathaliahalax@hotmail.com
}

RESUMO I INTRODUÇÃO: O abandono do tratamento da tuberculose (TB) auxilia no ciclo de propagação e contágio da doença, aumento dos custos, resistência medicamentosa e da morbimortalidade. Desta forma, este estudo buscou descrever os fatores de risco para o paciente de TB abandonar o tratamento, segundo a literatura nacional e internacional. METODOLOGIA: Trata-se de uma revisão integrativa da literatura, realizada nas bases de dados da Literatura Latino-Americana e do Caribe em Ciências da Saúde (LILACS) e Literatura Internacional em Ciências da Saúde (MEDLINE), a partir dos artigos científicos completos, publicados no período entre 2007 a 2017, em open acess, nos idiomas português, inglês e espanhol, bem como aqueles que abordassem nos resultados os fatores de risco para o abandono ao tratamento da TB. RESULTADOS: Foram encontrados 145 artigos científicos, dos quais 125 foram excluídos por não atenderem os critérios previamente estabelecidos, totalizando 20 artigos científicos para serem lidos e analisados na íntegra. Verificou-se que os aspectos sociodemográficos (uso de drogas, idade, sexo masculino, baixa escolaridade e renda, área rural, acessibilidade e estigma da doença), clínicos (coinfecção TB/HIV, abandono prévio, forma clínica extrapulmonar, comorbidades) e do tratamento (fase inicial da terapia, efeitos adversos, melhora sintomática) contribuem para o abandono do tratamento. CONCLUSÃO: Esta revisão aponta a necessidade de identificar precocemente os pacientes de TB vulneráveis ao desfecho, para então, utilizar as estratégias disponíveis, a exemplo do tratamento diretamente observado, visando garantir a adesão e, consequentemente, a cura.

\begin{abstract}
INTRODUCTION: The abandonment of tuberculosis (TB) treatment assists in the spread and infection of the disease, increasing costs, drug resistance and morbidity and mortality. In this way, this study sought to describe the risk factors for TB patient to abandon the treatment, according to the national and international literature. METHODOLOGY: It is an integrative literature review, held in the databases of the Latin American literature and Caribbean Health Sciences (LILACS) and International Health Sciences Literature (MEDLINE), from scientific articles, published in the period from 2007 to 2017 , in open access, in the languages Portuguese, English and Spanish, as well as those who approached the results to the risk factors for the abandonment to the treatment of TB. RESULTS: 145 scientific papers were found, of which 125 were excluded because they did not meet the previously established criteria, totaling 20 scientific articles to be read and analyzed in their entirety. The socio-demographic aspects (drug use, age, male sex, low schooling and income, rural area, accessibility and stigma of disease), clinical (TB / HIV co-infection, previous abandonment, extra pulmonary clinical form, comorbidities) and treatment (initial phase of therapy, adverse effects, symptomatic improvement) contribute to treatment withdrawal. CONCLUSION: This review points out the need to identify early TB patients vulnerable to closure, for then, use the strategies available, directly observed treatment, aiming to ensure adherence and, consequently, the cure.
\end{abstract}

DESCRIPTORS: Tuberculosis. Patient dropouts. Risk factors. Medication adherence.

DESCRITORES: Tuberculose. Pacientes desistentes do tratamento. Fatores de risco. Adesão à medicação. 


\section{Introdução}

A tuberculose (TB) é uma doença de âmbito mundial e um grave problema de saúde pública, tendo em vista que, em 2015, foram notificados aproximadamente 10,4 milhões de casos novos da doença, ocasionando 1,8 milhões de óbitos em todo o mundo, principalmente em países subdesenvolvidos, superando o HIV e malária juntos'.

As vulnerabilidades sociais, coinfecção TB/HIV e a resistência aos medicamentos agravam ainda mais esse cenário, aumentando o coeficiente de incidência e a probabilidade de desfechos desfavoráveis ${ }^{2}$, a exemplo do abandono do tratamento da TB, o qual auxilia no ciclo de propagação e contágio da doença, aumento dos custos, resistência medicamentosa e da morbimortalidade ${ }^{3}$.

No Brasil, estima-se que até 2035 seriam evitados 7.092 óbitos por TB em virtude da diminuição progressiva do abandono do tratamento ${ }^{2}$. No entanto, o que se observou no ano de 2015 é que a doença permanece com desfechos negativos, com baixa taxa de cura $(74,2 \%)$ e elevada para o abandono $(11 \%)$, as quais estão abaixo das metas preconizadas pela Organização Mundial da Saúde (OMS), de pelo menos $85 \%$ e até $5 \%$, respectivamente 2 .

Além disso, o país faz parte de um grupo de 30 países responsáveis por $80 \%$ da carga de TB no mundo', registrando em 2015, 69 mil casos novos da doença com uma taxa de incidência estipulada em 34,2 casos/ 100 mil hab., evidenciando que o país ainda não conseguiu alcançar as metas estabelecidas pela OMS como política até 2035 (menos de 10 casos de TB/ 100 mil hab.) $)^{2}$.

Diante disso, com o intuito de garantir a adesão ao tratamento e controle da doença, a OMS estabelece a estratégia Directly Observed Treatment ShortCourse (DOTS), tendo como um de seus pilares o Tratamento Diretamente Observado (TDO) ${ }^{4}$. Porém, a dificuldade de acesso e organização dos serviços de saúde, bem como a não corresponsabilização dos profissionais para detecção precoce dos casos, influenciam diretamente no retardo do diagnóstico e início do tratamento, contribuindo para a não adesão. dem desencadear tal desfecho, torna-se uma ferramenta essencial para o estabelecimento de ações e estratégias envolvendo a gestão, organização dos serviços e profissionais de saúde, visando a adesão e controle da doença. Diante disso, esta revisão tem como objetivo descrever os fatores de risco para o paciente de TB abandonar o tratamento, segundo a literatura nacional e internacional.

\section{Metodologia}

Trata-se de uma revisão integrativa da literatura, realizada em julho de 2017, a partir da questão norteadora: quais os fatores de risco para o paciente de TB abandonar o tratamento?

O levantamento foi realizado nas bases de dados da Literatura Latino-Americana e do Caribe em Ciências da Saúde (LILACS) e Literatura Internacional em Ciências da Saúde (MEDLINE), de acordo com os descritores indexados no Medical Subject Headings (MESH): "Tuberculose"/ "Tuberculosis", "Fatores de Risco"/ "Risk Factors", "Pacientes Desistentes do Tratamento"/ "Patient Dropouts" e "Adesão à Medicação"/ "Medication Adherence".

Para a busca bibliográfica, empregou-se a combinação entre os descritores através dos booleanos AND e OR. Desta forma, as expressões utilizadas foram (Tuberculose OR Tuberculosis) AND ("Fatores de risco" OR "Risk Factors") AND ("Adesão à Medicação" OR "Medication Adherence"), bem como (Tuberculose OR Tuberculosis) AND ("Fatores de risco" OR "Risk Factors") AND ("Pacientes Desistentes do Tratamento" OR "Patient Dropouts").

Os critérios de inclusão deste estudo foram artigos científicos completos, publicados no período entre 2007 a 2017, em livre acesso, nos idiomas português, inglês e espanhol, bem como aqueles que abordavam nos resultados sobre os fatores de risco para o abandono ao tratamento da TB. Foram excluídos os artigos duplicados, teses, dissertações, monografias, editoriais, artigos de revisão, cartas e manuais. 


\section{Resultados}

A partir da busca nas bases de dados, foram encontrados 145 artigos científicos, dos quais 23 estavam disponíveis no Lilacs e 122 no Medline. Destes, 125 foram excluídos por não atenderem aos critérios previamente estabelecidos, dos quais 1 não era artigo completo, 23 eram anteriores ao período previamente selecionado, 60 não estavam disponíveis em livre acesso, 28 não apresentavam resultados pertinentes ao objetivo desta revisão, uma vez que o foco do estudo era tratamento ou terapia antirretroviral, epidemiologia, diagnóstico, multirresistência, infecção tuberculosa latente, óbito, imigrantes, distribuição espacial e suporte tecnológico. Além disso, 8 estavam duplicados, 2 eram teses e 3 artigos de revisão. Diante disso, foram selecionados 20 artigos científicos ${ }^{3,5-23}$ para serem lidos e analisados na íntegra (Figura 1).

Figura 1. Etapas do processo de seleção de artigos para a revisão integrativa da literatura deste estudo

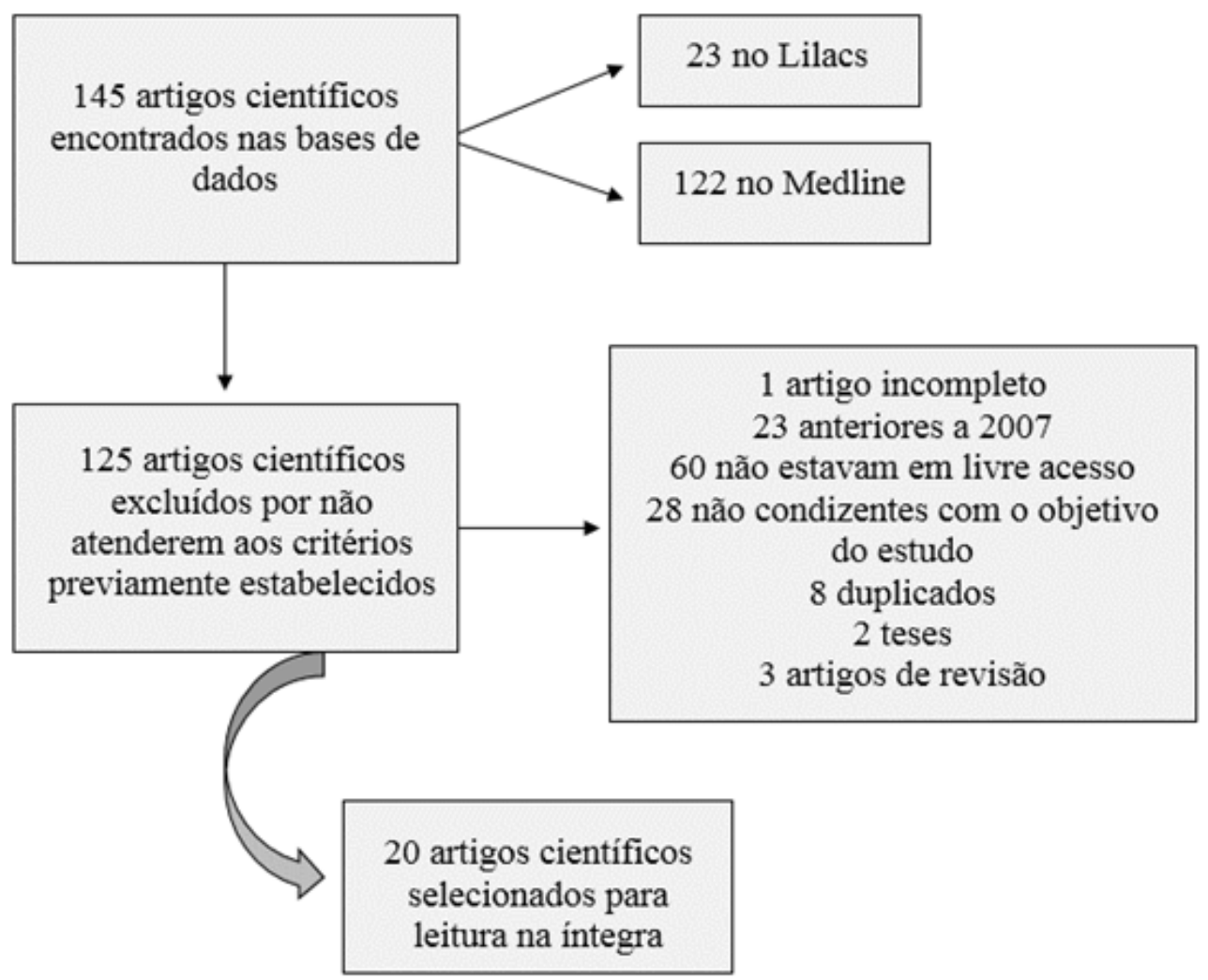

A maioria dos artigos estava em inglês (60\%), foram publicados em $2013(25 \%)$ e produzidos no cenário internacional $(60 \%)$. Já no Brasil, foram produzidos estudos nos estados Maranhão ${ }^{3}$, Rio de Janeiro ${ }^{5}$, Rio Grande do Sul6, Paraná ${ }^{7}$, Pernambuco ${ }^{8}$ e Minas Gerais ${ }^{9}$. Além disso, foram realizadas pesquisas multicêntricas em municípios brasileiros distintos (Manaus e Fortaleza ${ }^{10}$ ), e em países de fronteira (Brasil, Colômbia, Peru e Venezuela ${ }^{11}$ ). Quanto ao tipo de estudo, foram realizados predominantemente estudos caso-controle, em 8 artigos científicos (Quadro 1). 
Quadro 1. Distribuição dos artigos desta revisão por ano de publicação, país, idioma e tipo de estudo realizado.

\begin{tabular}{|c|c|c|c|}
\hline $\begin{array}{c}\text { Ano de } \\
\text { Publicação }\end{array}$ & País & Idioma & Tipo de estudo \\
\hline 2016 & Coréia & Inglês & Caso-controle ${ }^{12}$ \\
\hline 2016 & Etiópia & Inglês & Transversal 13 \\
\hline 2016 & Peru & Espanhol & $\begin{array}{l}\text { Caso-controle } \\
\text { retrospectivo }\end{array}$ \\
\hline 2015 & Argentina & Inglês & Transversal $^{15}$ \\
\hline 2015 & África do Sul & Inglês & Transversal ${ }^{16}$ \\
\hline 2015 & Etiópia & Inglês & Caso-controle ${ }^{17}$ \\
\hline 2014 & Brasil & Português & Transversal analítico ${ }^{3}$ \\
\hline 2013 & Brasil & Português & Transversal" \\
\hline 2013 & África do Sul & Inglês & Transversal $^{18}$ \\
\hline 2013 & Estados Unidos & Inglês & Série de $\operatorname{casos}^{19}$ \\
\hline 2013 & Estados Unidos & Inglês & Caso-controle 20 \\
\hline 2013 & República da Moldávia & Inglês & Coorte retrospectiva $^{21}$ \\
\hline 2012 & Brasil & Português & Caso-controle ${ }^{10}$ \\
\hline 2012 & Brasil & Português & Coorte 5 \\
\hline 2012 & Nigéria & Inglês & Coorte retrospectiva $^{22}$ \\
\hline 2011 & Brasil & Português & Caso-controle 6 \\
\hline 2011 & Quênia & Inglês & Caso-controle 23 \\
\hline 2010 & Brasil & Português & Transversal 7 \\
\hline 2007 & Brasil & Inglês & Coorte $^{8}$ \\
\hline 2007 & Brasil & Português & Caso-controle 9 \\
\hline
\end{tabular}

No que se refere aos fatores de risco para o abandono, identificou-se aspectos sociodemográficos, clínicos e do tratamento.

Quanto aos sociodemográficos, a maioria dos pacientes de TB faziam uso de drogas lícitas e/ou ilícitas (55\%), eram adultos jovens $(5 \%)$, homens $(30 \%)$, com baixa escolaridade $(30 \%)$, baixa renda $(25 \%)$, residentes em área rural (15\%), sem compreensão acerca da doença (15\%), com dificuldades de acesso aos serviços de saúde, analfabetos, sem moradia fixa e com estigma sobre a doença (10\%) (Quadro 2).

Além disso, no que diz respeito aos aspectos clínicos, a maioria dos que abandonaram o tratamento eram coinfectados TB/HIV (35\%), com história de abandono prévio ou reingresso após abandono $(25 \%)$, forma clínica extrapulmonar (15\%), culturas de escarro positivas após 2 meses de tratamento e presença de comorbidades (10\%) (Quadro 2).

E, para os fatores relacionados ao tratamento, os 3 primeiros meses de terapia $(30 \%)$, efeitos adversos dos medicamentos $(20 \%)$, melhora dos sinais e sintomas clínicos, tratamento hospitalar e regime de tratamento medicamentoso (10\%) contribuíram para o abandono (Quadro 2). 


\begin{tabular}{|c|c|c|}
\hline \multicolumn{2}{|r|}{ Fatores de Risco } & $\begin{array}{l}\mathrm{N}^{\circ} \text { de } \\
\text { artigos }\end{array}$ \\
\hline $\begin{array}{l}\text { FATORES } \\
\text { SOCIODEMOGRÁFICOS }\end{array}$ & $\begin{array}{l}\text { Uso de drogas lícitas e/ou ilícitas } \\
\text { Idade } \\
\text { Sexo masculino } \\
\text { Baixa escolaridade } \\
\text { Baixa renda ou classe econômica } \\
\text { Residência em área rural } \\
\text { Falta de informações sobre TB } \\
\text { Acesso aos serviços de saúde } \\
\text { Analfabetismo } \\
\text { Falta de moradia fixa ou morador de rua } \\
\text { Estigma da doença } \\
\text { Outros* }\end{array}$ & $\begin{array}{l}11 \\
10 \\
6 \\
6 \\
5 \\
3 \\
3 \\
2 \\
2 \\
2 \\
2 \\
10\end{array}$ \\
\hline $\begin{array}{l}\text { FATORES } \\
\text { CLÍNICOS }\end{array}$ & $\begin{array}{l}\text { Coinfecção TB/HIV } \\
\text { História de abandono prévio ou reingresso após abandono } \\
\text { TB extrapulmonar } \\
\text { Culturas positivas após } 2 \text { meses de terapia } \\
\text { Comorbidades } \\
\text { Outros* }\end{array}$ & $\begin{array}{l}7 \\
5 \\
3 \\
2 \\
2 \\
7\end{array}$ \\
\hline $\begin{array}{l}\text { FATORES DO } \\
\text { TRATAMENTO }\end{array}$ & $\begin{array}{l}\text { Fase inicial do tratamento } \\
\text { Efeitos adversos de medicamentos } \\
\text { Melhora dos sintomas clínicos } \\
\text { Tratamento hospitalar } \\
\text { Regime de tratamento } \\
\text { Outros* }\end{array}$ & $\begin{array}{l}6 \\
4 \\
2 \\
2 \\
2 \\
7\end{array}$ \\
\hline
\end{tabular}

* Outros - fatores de risco para o abandono do tratamento que foram citados uma única vez. Sociodemográficos (etnia não branca, doença mental, desemprego, alimentação inadequada, não morar com familiares, viver sozinho, falta de saneamento básico, chefe de família não possuir plano de saúde, não conseguir licença para realizar exames, encarcerados); clínicos (TB multidrogarresistente, tratamento prévio, razões médicas, doença cavitária, parceiro HIV positivo, lesão pulmonar extensa, esfregaço negativo no início do tratamento, não realização das baciloscopias de acompanhamento no segundo, quarto e sexto mês) e do tratamento (baixa pontuação em avaliação psicológica, uso de medicamentos à base de plantas, tratamento por um não pneumologista, organização dos serviços de saúde, terapia dupla, falta de pessoa de contato).

\section{Discussão}

\section{Preditores sociodemográficos para o abandono}

O uso de drogas lícitas e/ou ilícitas foi o mais mencionado. $O$ alcoolismo é citado em diversas literaturas como um fator clássico para os desfechos negativos do tratamento da $\mathrm{TB}^{6,8,23}$, no qual seu abuso implica no esquecimento da tomada dos medicamentos e da potencialização dos efeitos hepatotó$x i \cos ^{23}$. Além disso, a associação de uma ou mais drogas com o tabaco aumenta consideravelmente os riscos em relação à saúde ${ }^{18} \mathrm{e}$, consequentemente, no tratamento da doença.

O desfecho associado à idade reflete a proporção de adultos jovens acometidos pela TB que, normalmente são etilistas, não se alimentam de maneira adequada, não aceitam a doença, possuem uma relação de conflitos com o profissional ${ }^{3,6,7}$, bem como a presença de comorbidades ${ }^{13}$. 
Por outro lado, este fator merece um olhar atento visto ser uma classe produtiva e trabalhadora que, ao adoecer, perdem o vínculo empregatício ou até mesmo, morrem de maneira precoce ${ }^{3}$, o que pode refletir no desenvolvimento socioeconômico do local ou país em que residem ${ }^{13}$.

Além disso, a idade está associada ao sexo masculino, citado em diversos estudos $7,10,15,16,18,23$, no qual as demandas do trabalho e responsabilidade familiar dificultam a ida às consultas na unidade de saúde podendo levar à interrupção da medicação ou acompanhamento ${ }^{8,22}$.

Alguns autores trouxeram a combinação entre baixa escolaridade e baixa renda como fatores contribuintes para pouca adesão e abandono frequente do tratamento ${ }^{5,14}$. Dessa forma, baixo nível sociodemográfico relacionado à pobreza, a exemplo da educação e renda, evidencia o teor social da doença $a^{6,7}$.

Um estudo realizado em Recife revela que 0 analfabetismo gera fragilidades no que se refere ao acesso à informação, custos com transporte para o local de saúde e perdas de dias úteis no trabalho ${ }^{8}$. Diante disso, os profissionais de saúde devem ter um cuidado especial durante o tratamento com os pacientes que possuem baixa escolaridade ou analfabetos por meio da construção do vínculo, supervisão e incentivo à adesão ${ }^{7}$.

Ainda no que concerne ao baixo nível socioeconômico, os pacientes de TB que abandonam o tratamento, - fazem mediante a escolha de prioridades diante dos poucos recursos para atender suas demandas e suprir necessidades básicas, a exemplo da alimentação e transporte ${ }^{23}$, pelas restrições econômicas, bem como por estarem em um ambiente propício que não traz resultados positivos de saúde ${ }^{18}$.

De acordo com um estudo realizado em Belo Horizonte, o desemprego é associado ao desfecho pelo fato do indivíduo se preocupar com sua subsistência e adiar a procura pelo atendimento de saúde, diferentemente dos empregados que abandonam o tratamento pelo medo de perdê-lo", o que evidencia as vulnerabilidades as quais os pacientes de TB estão expostos.
Quanto ao local de residência nas áreas rurais, os doentes de TB podem ter menor conhecimento a respeito do tratamento da doença, o que se soma à distância de suas residências com o centro de saú$\mathrm{de}^{10,13}$, à falta de orientação a respeito da descentralização da assistência e da implementação do TDO?.

Dessa forma, para reverter este cenário, a expansão da Estratégia Saúde da Família (ESF) conjugado com o aumento da cobertura do TDO, transferência de casos de TB para a área urbana ${ }^{10,22}$, disponibilização de vale transporte para consulta e realização de exames pode influenciar na diminuição do abandono do tratamento.

Os fatores associados às condições de vida revelam que a saúde é influenciada pela desigualdade social no local de residência ${ }^{15}$. Segundo Bamrah e colaboradores (2013), em um estudo realizado nos Estados Unidos, os casos de abandono entre os pacientes de TB que não possuem moradia fixa ou que são moradores de rua, sugerem uma transmissão local com alta carga bacilar por conta das condições insalubres os quais estão submetidos, o que facilita o risco e a transmissão da doença entre essa população altamente móvel ${ }^{19}$.

A falta de informações sobre a TB sugere a necessidade, por parte dos profissionais de saúde, de uma educação sobre saúde pré-tratamento, explicações sobre a doença, seu tratamento, efeitos colaterais e a importância em cumprir todo o tratamento ${ }^{23}$, pois - maior conhecimento sobre a TB aumenta 0 empoderamento dos pacientes e os motiva a aderir o tratamento, tornando-os corresponsáveis pelo enfretamento de sua enfermidade ${ }^{9,12}$.

Por outro lado, fatores culturais e regionais estão associados ao não conhecimento da doença, como o fato de atribuir semelhança ao HIV/aids, o que contribui para o estigma da $\mathrm{TB}^{23}$. Devido a isso, alguns pacientes de TB preferem realizar o tratamento em um local distante de sua residência9, contribuindo para o desfecho do abandono.

A doença mental contribui para $\circ$ abandono, pois diante de uma condição crônica, tal como a TB, necessita de cuidados especiais frente às respostas desencadeadas nas pessoas do grupo social e fami- 
liar, desde as dificuldades cotidianas no cuidado do paciente até o desconhecimento da doença em $\mathrm{si}^{3}$.

\section{O abandono do tratamento associado a fatores clínicos}

A coinfecção TB/HIV foi responsável, em 2015, por $11 \%$ do total de casos de TB no mundo', o que corrobora os achados desta revisão que a traz como a mais citada dentre os fatores clínicos de risco. Estudo realizado por Muture e colaboradores (201 1), no Quênia, revela que 54,9\% dos pacientes eram coinfectados TB/HIV, o que os fazia ter mais custos com transporte por terem que frequentar clínicas separadas para o tratamento de ambas das enfermidades ${ }^{23}$.

Concomitante a isso, o aumento dos efeitos colaterais e carga de medicamentos para o tratamento duplo ${ }^{18,22}$, impacta 0 cotidiano destes pacientes que possuem demandas associadas ao trabalho e à família $^{18}$, configurando-se como um duplo desafio que pode contribuir para o desfavorável prognóstico e desfecho da doença ${ }^{6,17}$.

Desta forma, o planejamento de ações com os pacientes coinfectados TB/HIV, educação em saúde e ações de vigilância poderá auxiliar no acompanhamento e tratamento ${ }^{8}$, além de evitar a mortalidade destes $\operatorname{casos}^{5}$.

O histórico de abandono prévio ou reingresso após abandono são importantes para detectar o risco de um novo episódio de abandono ${ }^{12}$. Um estudo multicêntrico realizado em países de fronteira com o Brasil, afirma que tais indivíduos apresentam 3 vezes mais chance de não concluir o tratamento ${ }^{11}$, o que sugere a importância de completar o tratamento primário com acompanhamento e suporte adequado ${ }^{8}$.

Devido a uma tendência de tratar a forma clínica extrapulmonar por mais de 12 meses $^{20}$, o diagnóstico ser invasivo, baixa probabilidade de cultivo do bacilo e avaliação clínica ser incerta ${ }^{12}$, o abandono relacionado a estes casos extrapulmonares, sugere um atraso no diagnóstico e tratamento, o que possibilita que a doença se torne mais grave e, dessa forma, a cura seja mais difícil de ser alcançada ${ }^{8,13}$.
O abandono do tratamento está associado ao aumento das taxas de resistência medicamentosa ${ }^{16}$ não só pelo tratamento necessitar ser mais longo, mas também pelos efeitos colaterais de drogas mais tóxicas e, em alguns casos, ausência de melhora clínica $^{21}$, $\circ$ que aumenta $\circ$ risco de transmissão entre as pessoas que vivem e trabalham perto destes pacientes de $\mathrm{TB}^{18}$.

A realização das baciloscopias de controle mensal serve para o acompanhamento dos casos e redução em até 12 vezes do desfecho do abandono ${ }^{11}$, pois mediante resultado, permite o monitoramento do caso pelo profissional de saúde.

Neste sentido, culturas de escarro positivas após 2 meses de tratamento ${ }^{20}$ questiona-se de que forma e como é realizado o acompanhamento destes casos, tendo em vista que as medicações antituberculosas ocasionam respostas negativas se realizado um tratamento contínuo ${ }^{17}$.

\section{O tratamento como fator de risco para o abandono}

A fase inicial do tratamento corresponde ao acontecimento de uma rápida melhora sintomática ${ }^{12}$ pelo fato dos medicamentos reduzirem rapidamente a carga bacilar ${ }^{23}$. Neste sentido, os pacientes de TB abandonam 0 tratamento por acharem que estão curados pela melhora dos sinais e sintomas ${ }^{3,6,22}$, somado à falta de informação sobre este acontecimento ${ }^{23}$.

Diante disso, os profissionais de saúde devem promover orientações a respeito desta fase inicial do tratamento através de educação em saúde, e supervisionar os casos com potencial risco para o abandono por meio do suporte da equipe multiprofissio$\mathrm{nal}^{22}$.

De acordo com Orofino e colaboradores (2012), em um estudo realizado no Rio de Janeiro, a quantidade de medicamentos utilizados, em alguns casos, é consequência do abandono, visto alguns pacientes de TB estarem em retratamento por terem abandonado outras vezes ${ }^{5}$, o que contribui para o aumento da probabilidade de casos relacionados à resistência medicamentosa. 
Além disso, este fato pode ser decorrente dos efei-

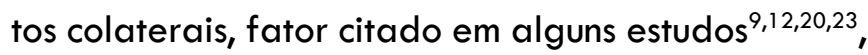
- que sugere uma reavaliação de novos fármacos que sejam mais eficazes e menos tóxicos ${ }^{20}$.

A supervisão da ingesta medicamentosa e monitoramento dos casos até a cura é uma das propostas do TDO ${ }^{4}$. Por conta disso, estudos mostram que essa estratégia preconizada pela OMS, auxilia na adesão e sucesso do tratamento ${ }^{13,17,20}$. No entanto, em um estudo realizado na África do Sul, mesmo os pacientes de TB sendo acompanhados por meio do TDO, a taxa de abandono foi alta (30\%), o que sugere que outros fatores estejam associados ao desfecho ${ }^{16}$, a exemplo da fragilidade do vínculo e acolhimento entre profissional-usuário-família.

Dessa forma, conhecer os fatores de risco auxilia de forma positiva e construtiva nos programas de controle da doença e no desenvolvimento de estratégias para os pacientes de TB com maior risco para o abandono ${ }^{19}$.

\section{Considerações finais}

Este estudo permitiu identificar os fatores sociodemográficos, clínicos e do tratamento que, associados ou não, contribuem para um desfecho desfavorável, como o abandono do tratamento.

Neste sentido, verificou-se uma carência de estudos nacionais que abordem a temática, o que revela uma lacuna no que diz respeito à produção científica em locais subdesenvolvidos que trazem a TB como uma verdadeira epidemia. Diante disso, conhecer os fatores de risco para o abandono em âmbito nacional torna-se um desafio para o controle da doença no país.

Mediante o exposto, esta revisão aponta a necessidade de identificar precocemente os pacientes de TB vulneráveis ao desfecho, para então, utilizar as estratégias disponíveis, a exemplo do TDO, para garantir a adesão e, consequentemente, a cura.

\section{Agradecimentos}

Agradecimentos ao Centro de Estudo e Pesquisa em Saúde Coletiva (CEPESCO) da Universidade Federal de Rondônia (UNIR) e ao Conselho Nacional de Desenvolvimento Científico e Tecnológico (CNPq) pelo financiamento de bolsa de iniciação científica (PIBIC 2016-2017).

\section{Contribuições dos autores}

MRL Ferreira trabalhou na concepção, análise e interpretação dos resultados, redação do artigo e revisão crítica. RO Bonfim, TC Siqueira e NH Orfão contribuíram na redação do artigo. Todos os autores aprovaram a versão final escrita a ser publicada.

\section{Conflitos de interesses}

Nenhum conflito financeiro, legal ou político envolvendo terceiros (governo, empresas e fundações privadas, etc.) foi declarado para nenhum aspecto do trabalho submetido (incluindo mas não limitandose a subvenções e financiamentos, conselho consultivo, desenho de estudo, preparação de manuscrito, análise estatística, etc).

\section{Referências}

1. World Health Organization. Global tuberculosis report 2016. Geneva: World Health Organization; 2016.

2. Brasil. Ministério da Saúde. Secretaria de Vigilância em Saúde. Perspectivas brasileiras para o fim da tuberculose como problema de saúde pública. Boletim Epidemiológico. 2016;47(13):1-15.

3. Silva PF, Moura GS, Caldas AJM. Fatores associados ao abandono do tratamento da tuberculose pulmonar no Maranhão, Brasil, no período de 2001 a 2010. Cad Saúde Pública. 2014;30(8): 1745-1754. doi: 10.1590/0102$\underline{311 \times 00124513}$

4. Brasil. Ministério da Saúde. Secretaria de Vigilância em Saúde. Departamento de Vigilância Epidemiológica. Manual de Recomendações para o Controle da Tuberculose no Brasil. Brasília: Ministério da Saúde; 2011.

5. Orofino RL, Brasil PEA, Trajman A, Schmaltz CAS, Dalcolmo $M$, Rolla VC. Preditores dos desfechos do tratamento da tuberculose. J Bras Pneumol. 2012;38(1):88-97. doi: $10.1590 /$ S1806-37132012000100013

6. Campani STA, Moreira JS, Tietbohel CN. Fatores preditores para o abandono do tratamento da tuberculose pulmonar preconizado pelo Ministério da Saúde do Brasil na cidade de Porto Alegre (RS). J Bras Pneumol. 2011 ; 37(6):776-782. doi: $10.1590 /$ S1806-37132011000600011

7. Giroti SKO, Belei RA, Moreno FN, Silva FS. Perfil dos pacientes com tuberculose e os fatores associados ao abandono do tratamento. Cogitare Enferm. 2010;15(2):271 77. doi: $10.5380 /$ ce.v15i2.17860 
8. Albuquerque MFPM, Lucena-Silva N, Dantas AT, Dantas OMS, Ximenes RAA, Rodrigues $L C$, et al. Factors associated with treatment failure, dropout, and death in a cohort of tuberculosis patients in Recife, Pernambuco State, Brazil. Cad Saude Publica. 2007;23(7):1573-1582.

doi: $10.1590 /$ S0102-311 2007000700008

9. Paixão LMM, Gontijo ED. Perfil de casos de tuberculose notificados e fatores associados ao abandono, Belo Horizonte, MG. Rev Saúde Pública. 2007;41 (2):205-213. doi: $10.1590 /$ S0034-89102007000200006

10. Braga JU, Pinheiro JS, Matsuda JS, Barreto JAP, Feijão AMM. Fatores associados ao abandono do tratamento da tuberculose nos serviços de atenção básica em dois municípios brasileiros, Manaus e Fortaleza, 2006 a 2008. Cad Saúde Colet. $2012 ; 20(2): 225-233$.

11. Belo EN, Orellana JDY, Levino A, Basta PC. Tuberculose nos municípios amazonenses da fronteira Brasil-ColômbiaPeru-Venezuela: situação epidemiológica e fatores associados ao abandono. Rev Panam Salud Publica. $2013 ; 34(5): 321-9$.

12. Park CK, Shin HJ, Kim YI, Lim SC, Yoon JS, Kim YS, et al. Predictors of Default from Treatment for Tuberculosis: a Single Center Case-Control Study in Korea. J Korean Med Sci. 2016; 31 (2):254-260. doi: 10.3346/ikms.2016.31.2.254

13. Gebrezgabiher G, Romha G, Ejeta E, Asebe G, Zemene E, Ameni G. Treatment Outcome of Tuberculosis Patients under Directly Observed Treatment Short Course and Factors Affecting Outcome in Southern Ethiopia: A Five-Year Retrospective Study. PLoS ONE 11 (2):e0150560. doi: 10.1371 /iournal.pone.0150560

14. Anduaga-Beramendi A, Maticorena-Quevedo J, Beas R, Chanamé-Baca DM, Veramendi $M$, Wiegering-Rospigliosi $A$, et al. Factores de riesgo para el abandono del tratamiento de tuberculosis pulmonar sensible en un establecimiento de salud de atención primaria, Lima, Perú. Acta Med Peru. 2016; $33(1): 21-8$.

15. Herrero MB, Arrossi S, Braga JU, Ramos S. Social determinants of nonadherence to tuberculosis treatment in Buenos Aires, Argentina. Cad Saúde Pública. 2015;31(9): 1983-1994. doi: 10.1590/0102-311X00024314

16. Moyo S, Cox HS, Hughes J, Daniels J, Synman L, De Azevedo V, et al. (2015) Loss from Treatment for Drug Resistant Tuberculosis: Risk Factors and Patient Outcomes in a Community-Based Program in Khayelitsha, South Africa. PLoS ONE. 2015;10(3):e0118919. doi: 10.1371 /iournal.pone.0118919

17. Amante TD, Ahemed TA. Risk factors for unsuccessful tuberculosis treatment outcome (failure, default and death) in public health institutions, Eastern Ethiopia.

The Pan African Medical Journal. 2015;20:247.

doi: 10.11604 /pami.2015.20.247.3345
18. Naidoo P, Peltzer K, Louw J, Matseke G, McHunu G, Tutshana B. Predictors of tuberculosis (TB) and antiretroviral (ARV) medication non-adherence in public primary care patients in South Africa: a cross sectional study. BMC Public Health. 2013; 26(13):396. doi: $10.1186 / 1471-2458-13-396$

19. Bamrah S, Woodruff RSY, Powell K, Ghosh S, Kammerer JS, Haddad MB. Tuberculosis among the homeless, United States, 1994-2010. Int J Tuberc Lung Dis. 2013; 17(1 1):1414-1419. doi: $10.5588 /$ iitld.13.0270

20. Pettit AC, Cummins J, Kaltenbach LA, Sterling TR, Warkentin JV. Non-adherence and drug-related interruptions are risk factors for delays in completion of treatment for tuberculosis. Int J Tuberc Lung Dis. 2013; 17(4):486-492. doi: $10.5588 /$ iitld. 12.0133

21. Jenkins HE, Ciobanu A, Plesca V, Crudu V, Galusca I, Soltan V, et al. Risk factors and timing of default from treatment for non-multidrug-resistant tuberculosis in Moldova. Int J Tuberc Lung Dis. 2013;17(3):373-380. doi: 10.5588 /ijtld. 12.0464

22. Ifebunandu NA, Ukwaja KN. Tuberculosis treatment default in a large tertiary care hospital in urban Nigeria: prevalence, trend, timing and predictors. J Infect Public Health. 2012; 5(5):340-345. doi: $10.1016 /$ i.jiph.2012.06.002

23. Muture BN, Keraka MN, Kimuu PK, Kabiru EW, Ombeka VO, Oguya F. Factors associated with default from treatment among tuberculosis: patients in Nairobi province, Kenya: a case control study. BMC Public Health. $2011 ; 11: 696$. doi: 10.1186/1471-2458-11-696 